\title{
Multistage Relay Network Topology using IEEE802.11ax for Construction of Multi-robot Environment
}

\author{
Ryo Odake, Kei Sawai, Noboru Takagi, Hiroyuki Masuta, Tatsuo Motoyoshi \\ Graduate School of Engineering, Toyama Prefectural University \\ Toyama, Japan
}

\begin{abstract}
This paper describes an information gathering system comprising multiple mobile robots and a wireless sensor network. In general, a single robot searches an environment using a teleoperation system in a multistage relay network while maintaining communication quality. However, the search range of a single robot is limited, and it is difficult to gather comprehensive information in large-scale facilities. This paper proposes a multistage relay network topology using IEEE802.11ax for information gathering by multi-robot. In this multi-robot operation, a mobile robot carries wireless relay nodes and deploys them into the environment. After a network is constructed, each robot connects to this network and gathers information. An operator then controls each robot remotely while monitoring its end-to-end communication quality with each mobile robot in the network. This paper proposes a method assuming the end-to-end throughput with multiple mobile robots. The validity of the proposed method is then inspected via an evaluation experiment on multi-robot teleoperation. The experimental results show that the network constructed with the proposed topology is capable of maintaining the communication connectivity of more than three mobile robots.
\end{abstract}

Keywords-Multi-robot system; IEEE802.11ax; information gathering; multistage relay network; network topology

\section{INTRODUCTION}

After a disaster, disaster reduction activities are performed in the affected area to prevent the damage from spreading. In the implementation of disaster reduction activities, information should be gathered to determine the damage status [1-2]. Helicopter aerial photographs, existing infrastructure (such as surveillance cameras), and rescue teams can be used to gather such information [3-6]. However, in enclosed spaces, such as tunnels and underground malls, it is difficult to gather information using helicopters. In some cases, existing infrastructure cannot be used due to malfunction or lack of power supply. Moreover, there is a risk of injuring humans or inducing secondary disasters during information gathering by rescue teams. Therefore, the use of mobile robots is widely considered to gather information in enclosed spaces after disasters [7-11].

Two communication methods are adopted for mobile robots: wired and wireless. Wired communication maintains a stable communication quality and power supply from cables [12]. However, cables may be disconnected and communication with the mobile robots can be interrupted when cables become tangled with obstacles or the wheels of the mobile robot. Wireless communication offers high runability, as there is no physical restriction by cables [13-14]. However, in wireless communication, mobile robots may become isolated when radio waves are hindered by obstacles. Therefore, in an enclosed space after a disaster, it is necessary to use the communication method that best matches the purpose and the situation of the disaster area [15-16]. This paper discusses a method for gathering information using wireless communication in environments where it is difficult to operate a mobile robot using wired communication.

Robot wireless sensor networks (RWSNs) involve the teleoperation of mobile robots using wireless communication [17-19]. In an RWSN system, a mobile robot expands its search range by deploying a relay node called a sensor node (SN) in its path (Fig. 1). In a network using a multistage relaying such as RWSNs, the communication quality decreases as the number of relays and the distance between nodes increase. Therefore, it is difficult to maintain the communication connectivity of mobile robots in a multistage relay network, and single robots are mainly operated. Moreover, the search range of a single robot in a large-scale facility is limited. This paper proposes a multistage relay network topology for constructing a multi-robot environment. The experiment in this paper constructed a multistage relay network with the proposed topology and measured the communication quality to confirm the validity of the proposed topology for constructing a multi-robot environment. This experiment used a bandwidth compression throughput to measure the communication quality at the packet level.

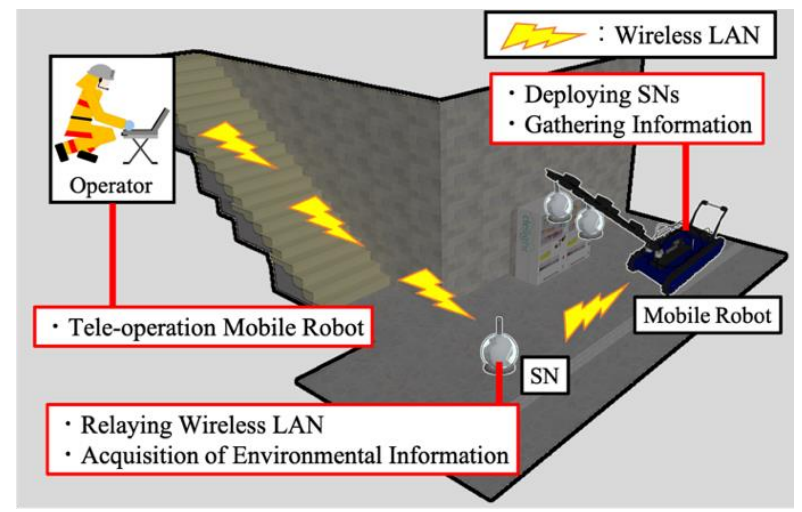

Fig. 1. RWSN. 


\section{TELEOPERATION METHOD FOR MOBILE ROBOTS USING MULTISTAGE RELAY NETWORK}

In the teleoperation of mobile robots by wireless communication, the search range is limited due to radio-wave damping. Therefore, wireless communication is not effective for gathering information in large-scale facilities. An RWSN relays SNs to expand the search range of a mobile robot. It also extends the network construction range by teleoperating the mobile robot with each node relayed in advance (Fig. 2). This allows the operator to teleoperate the mobile robot without the risk of a decrease in communication quality due to the increase in the number of relays. An RWSN deploys SNs in consideration of the communication quality between nodes, thereby constructing the network flexibly according to the environment. Therefore, RWSNs have scalability and flexibility, which make them effective for searching in environments with many obstacles.

However, in a multistage relay network, such as an RWSN, packets are processed for transfer within the SN. Hence, the transmission rate decreases and the packet reception interval become misaligned as the number of relays and the distance between nodes increase. Consequently, the mobile robot becomes less operable; it can become isolated due to moving out of the communication range by mistake or failing to maintain communication connectivity. Therefore, in an RWSN, it is common to operate a single robot to maintain the communication quality required for its teleoperation with the mobile robot. However, a single robot encounters limitations in searching and gathering comprehensive information in largescale facilities. Given the importance of multi-robot systems for solving the problem of using RWSNs in large-scale facilities, and the paper aims to construct a multi-robot environment using a multistage relay network. The next section shows the flow of the multi-robot system using a multistage relay network and the requirements for the network to teleoperate multiple robots.

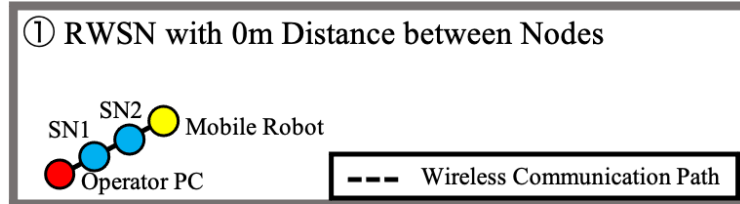

(2) Increase Distance between Operator PC and SN1

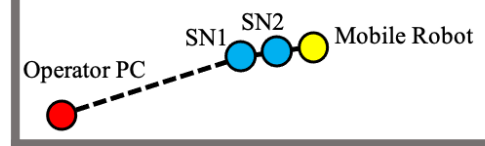

(3) Increase Distance between SN1 and SN2

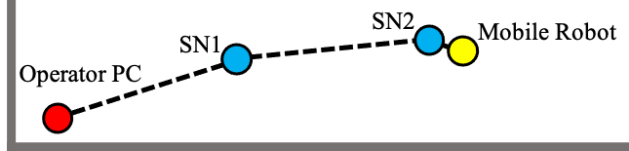

(4) Increase Distance between SN2 and Mobile Robot

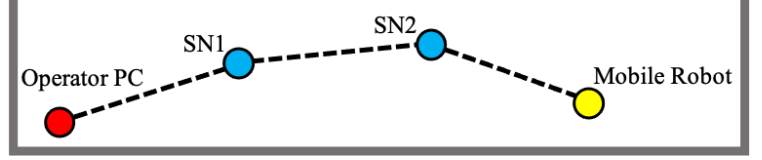

Fig. 2. Increasing Distance between Nodes while Maintaining Communication Connectivity in RWSNs.

\section{ReQuirements OF Multistage Relay NeTwORK}

Let mobile robot $\langle 1\rangle$ be the mobile robot that deploys the $\mathrm{SN}$. The flow from the construction of the network to the operation of the mobile robots is shown in Fig. 3.

- The construction range of a multistage relay backbone network is expanded with the mobile robot $\langle 1\rangle$ as shown in Fig. 2.

- Multiple mobile robots are connected to the constructed backbone network.

- Mobile robot $\langle 1\rangle$ and the other mobile robots search within the network construction range while switching the nodes to be connected.

The requirements of the multistage relay backbone network for multi-robot operation are as follows:

1) Remote control of a total of three or more mobile robots

2) A throughput of $20.0 \mathrm{Mbps}$ or higher in the communication path between each operator PC and each mobile robot.

About three mobile robots with five cameras each are needed to search $100 \mathrm{~m}$ of an enclosed space. Therefore, in requirement (1) set the number of teleoperated mobile robots to three. Moreover, the mobile robot used for searching in the nuclear power plant damaged by the Great East Japan Earthquake gathered information based on five camera images and required a throughput of $20.0 \mathrm{Mbps}$. Therefore, in requirement (2), the throughput required for the teleoperation of a single mobile robot is set to be $20.0 \mathrm{Mbps}$ or higher.

Many wireless teleoperation systems for mobile robots are based on TCP/IP protocols. TCP/IP is highly suitable for the communication of mobile robots because most of the control system of a mobile robot involves a PC. Therefore, socket communication is often adopted for mobile robot communication, and information communication by packet transmission and reception is typical. Therefore, RWSNs adopt wireless LAN as their communication method. However, the theoretical value of IEEE $802.11 \mathrm{~b} / \mathrm{g}$ used in RWSNs is 54 Mbps, which is an insufficient throughput for multi-robot operation. This study constructs a network using IEEE802.11ax, which has a theoretical value of about 1,200 Mbps. The next section describes the proposed multistage relay network topology, which takes into account the abovementioned requirements.

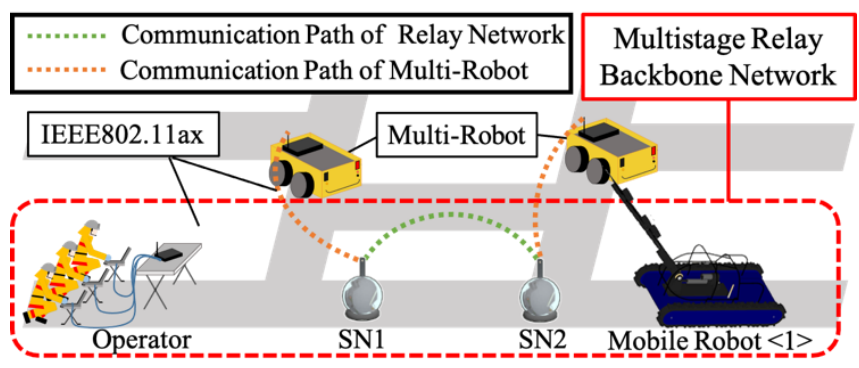

Fig. 3. Gathering Information by Multi-robot using Multistage Relay Network. 


\section{PROPOSED NeTwORK TOPOLOGY}

A network topology is a form of connection that shows how devices such as operator PCs are connected to each other. From the flow of the multi-robot operation in Section 3, the multistage relay part of the proposed topology should be a static network and an RWSN to prevent any change in communication quality due to the dynamic communication path change. The proposed network topology for a multi-robot system is shown in Fig. 4. The devices of each node are in Fig. 5, and Fig. 6 depicts how the nodes are connected. In this network topology, each node in the multistage relay network is equipped with an access point (AP) and an adapter device; this creates a communication path between the operator PC and the mobile robot $\langle 1\rangle$. Then, the multi-robots are equipped with adapter devices, which are connected to the APs installed in each node of the multistage relay network to construct a communication path between each operator PC and each mobile robot. In this topology, a network is constructed for each node, and each robot can comprehensively search within the network construction range while switching the AP to which it is connected.

In case of SN failure or battery power insufficiency, it is necessary to replace the $\mathrm{SN}$ and reconstruct the multistage relay network. In this topology, the communication path between the operator $\mathrm{PC}$ and mobile robot $\langle 1\rangle$ can be reconstructed with the same topology as that before the communication interruption by connecting the newly deployed $\mathrm{SN}$ to the AP of the node on the upload side that is still functioning.

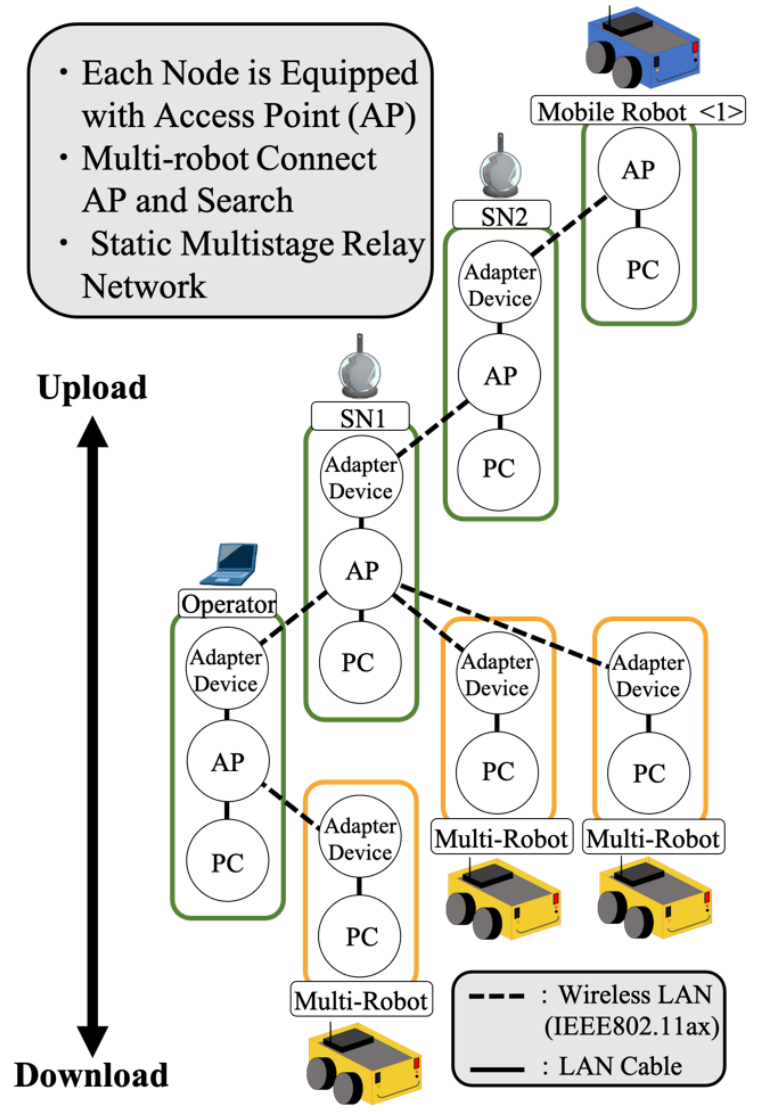

Fig. 4. Proposed Topology for Constructing a Multi-robot Environment.

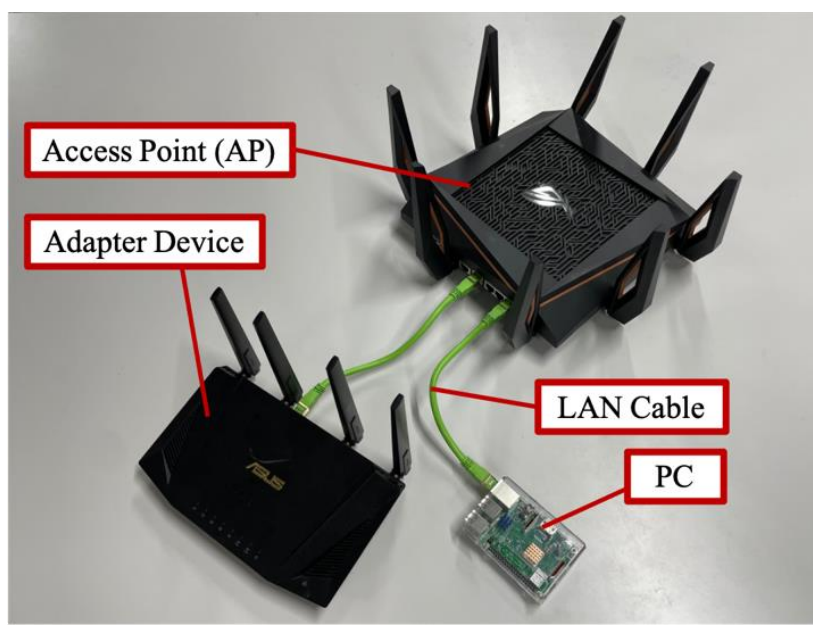

Fig. 5. Device of a Node Constituting the Multistage Relay Network.

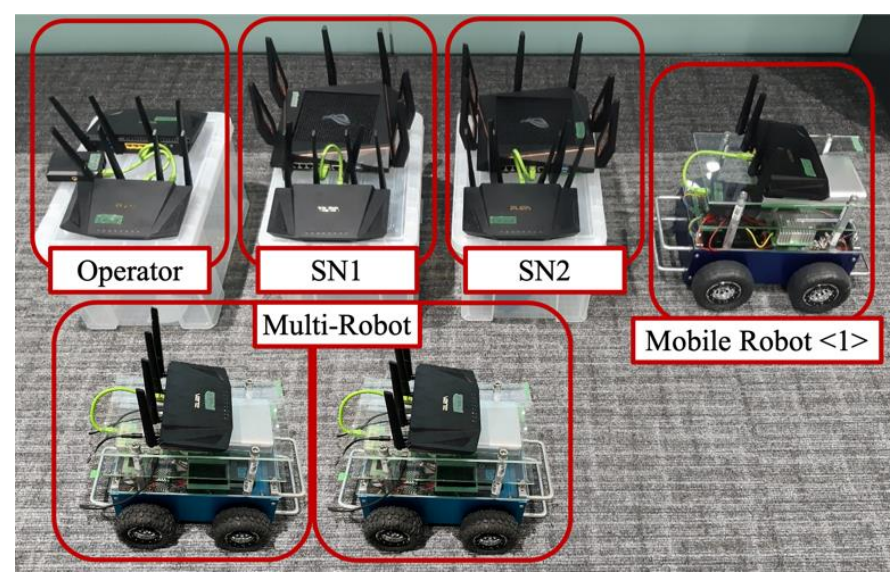

Fig. 6. Multistage Relay Network and Robots.

\section{Evaluation OF COMMUNiCATION QUALITY USING BANDWIDTH COMPRESSION THROUGHPUT MEASUREMENT}

This section define the communication quality characteristics that should be evaluated when teleoperating a mobile robot and explain how to measure them.

The operator receives packets containing camera and sensor information acquired by the mobile robots, and teleoperates the mobile robots (Fig. 7). Therefore, the transmission speed between the operator PC and the mobile robots should be monitored to maintain stability of the teleoperation of the mobile robots. This paper evaluates the transmission speed in a TCP/IP-compliant communication path as the throughput at the packet level. Throughput (bps) indicates the transmission speed received by the receiving PC per second. This system is expected to send a large amount of sensed information from the mobile robots. Therefore, this paper uses a band compression throughput measurement that can determine the upper limit of the transmission speed.

Bandwidth compression throughput represents the maximum number of data receivable per second by transmitting a large number of measurement packets from the transmitting side. Additionally, this measurement method send as many packets as the computer can process to measure the upper limit of the throughput accurately. The packet size of the 
transmission packet is set to 1,400 bytes, which is the upper limit. In this system, the bandwidth compression throughput is calculated assuming that the packet size is $B$ (byte), the total number of received packets is $n$, and the time required to complete the measurement is $t(\mathrm{~s})$. Then, the band compression throughput, Th (bps), can be expressed by the following equation:

$T h=\frac{8 B n}{t}$

\section{Tele-operation of Mobile Robot}

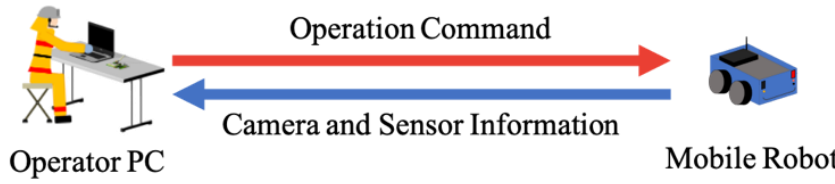

\section{Bandwidth Compression Throughput Measurement}

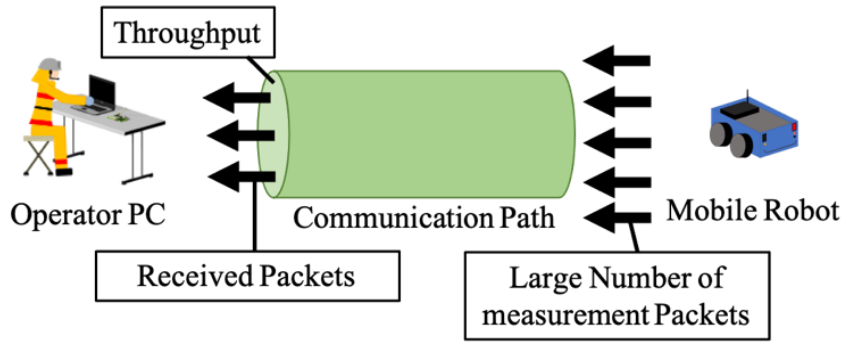

Fig. 7. Evaluation of Communication Quality of Teleoperation of Mobile Robots.

\section{Possibility OF CONSTRUCTING A Multi-Robot ENVIRONMENT WITH PROPOSED MULTISTAGE RELAY NETWORK}

\section{A. Experiment on Increasing Distance between Nodes}

This experiment examined the change in throughput with increasing distance between nodes to confirm whether such distance can be increased during network construction. This experiment measured the bandwidth compression throughput to determine the change in throughput with increasing distance between nodes. The distance between $\mathrm{SN} 1$ and $\mathrm{SN} 2$ was increased from $0 \mathrm{~m}$ to $5 \mathrm{~m}$ at $5 \mathrm{~m}$ intervals until communication was lost, and the bandwidth compression throughput was measured 10 times in both directions at each point. Then, this experiment loaded the network by sending 50,000 packets of 1,400 bytes. The upload direction was from $\mathrm{SN} 1$ to SN2, and the download direction was from SN2 to $\mathrm{SN} 1$, as shown in Fig. 4. A straight, paved $250 \mathrm{~m}$ road was used (Fig. 8). Furthermore, this experiment used a Raspberry Pi 4 Model B as the PC, ASUS RT-AX3000 as the router, and a CAT8 LAN cable.

Fig. 9 and 10 show the experimental results in the upload and download directions, respectively. The figures state the minimum, average, and maximum throughput values at each point. After the bandwidth compression throughput measurement in the download direction at the $230 \mathrm{~m}$ point, SN1 lost connection to the AP of SN2, so the upstream direction could be measured to the $225 \mathrm{~m}$ point and the download direction to the $230 \mathrm{~m}$ point. Thus, the distance between nodes can be extended, so this experiment constructed a multi-robot environment with a multistage relay network, as explained in the next section.

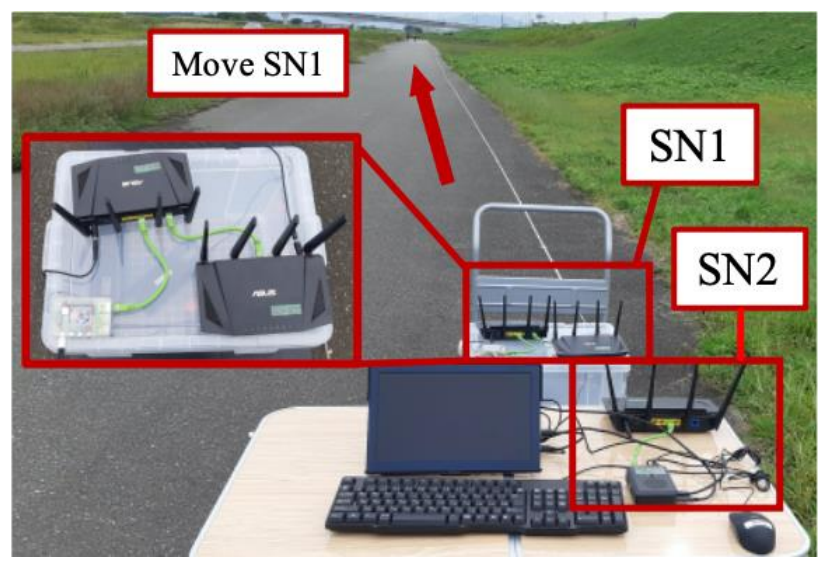

Fig. 8. Experimental Environment; Increase in Distance between Nodes.

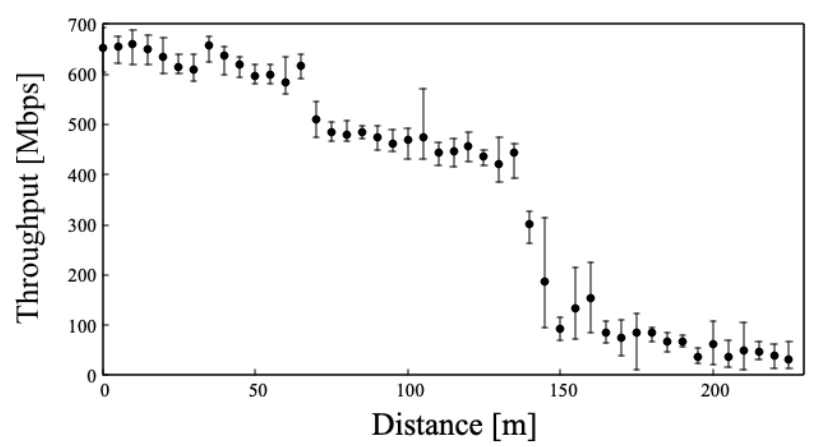

Fig. 9. Throughput of Upload; Increase in Distance between Nodes.

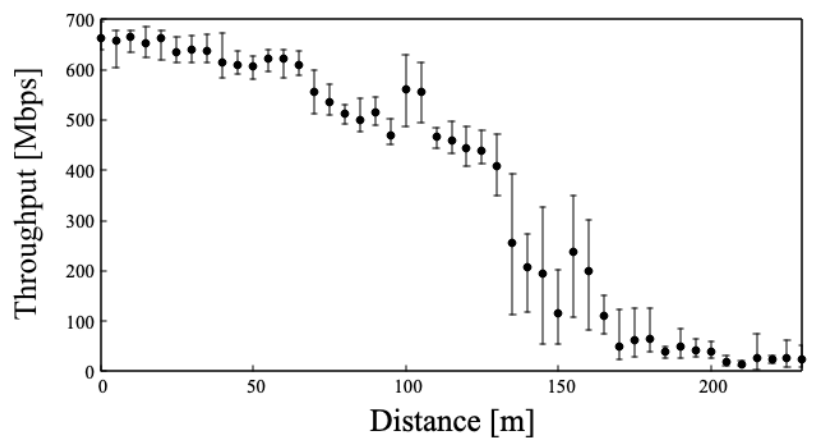

Fig. 10. Throughput of Download; Increase in Distance between Nodes.

\section{B. Construction of Multi-robot Environment using Proposed Multistage Relay Network.}

The purpose of this experiment is to confirm whether it is possible to construct a multi-robot environment that satisfies the requirements of the proposed network topology. This experiment measured the bandwidth compression throughput to confirm whether the constructed network can maintain the throughput required for multi-robot teleoperation. First, the distance between each node between the operator PC and mobile robot $\langle 1\rangle$ in the proposed topology was increased from $0 \mathrm{~m}$ to $90 \mathrm{~m}$ at $10 \mathrm{~m}$ intervals. Then the bandwidth compression throughput was measured five times in both directions between the operator $\mathrm{PC}$ and the mobile robot $<1>$ at 
each point. A mobile robot was connected to the constructed network (the distance between each node was $90 \mathrm{~m}$ ) and teleoperated, and bandwidth compression throughput measurement was performed five times in both directions between the operator PC and the mobile robot every $10 \mathrm{~m}$. This experiment used a Raspberry Pi 4 Model B as the PC, ASUS RT-AX3000 and GT-AX11000 as the router, and a CAT8 LAN cable.

Fig. 11 shows the experimental environment of this section. This experiment was performed indoors with a straight $90 \mathrm{~m}$ line, so the operator PC and SN2 were deployed at the $0 \mathrm{~m}$ point, and SN1 and mobile robot $\langle 1>$ were moved to increase the distance between each terminal in the fold. A mobile robot connected to the constructed network switched the nodes to which it was connected is the following manner:

- The mobile robot was teleoperated by connecting to the operator's AP from the $0 \mathrm{~m}$ point to the $90 \mathrm{~m}$ point (point where SN1 was deployed).

- The mobile robot was teleoperated by connecting to the AP of SN1 from the $90 \mathrm{~m}$ point to the $180 \mathrm{~m}$ point (point where SN2 was deployed).

- The mobile robot was teleoperated by connecting to the AP of SN2 from the $180 \mathrm{~m}$ point to the $270 \mathrm{~m}$ point (point where mobile robot $<1>$ was deployed).

Fig. 12 and 13 show the throughput values between the operator $\mathrm{PC}$ and mobile robot $<1>$ during the expansion of the network construction range. Fig. 14 and 15 show the throughput values between the operator PC and the mobile robot during the teleoperation of the mobile robot. Fig. 12-14 shows the minimum, average, and maximum throughput values at each point. As stated in Fig. 11 and 12, the range of the multistage relay network was expanded to a distance of $90 \mathrm{~m}$ between each node ( $270 \mathrm{~m}$ in total), and the throughput was maintained at more than $60 \mathrm{Mbps}$ in both directions. Fig. 13 and 14 also show that the throughput between the operator PC and the mobile robot remained over $60 \mathrm{Mbps}$.

These results indicate that a multistage relay network constituted by the proposed topology can maintain the required throughput for multi-robot teleoperation. Therefore, the robots connected to the multistage relay network constructed in this experiment could operate stably.

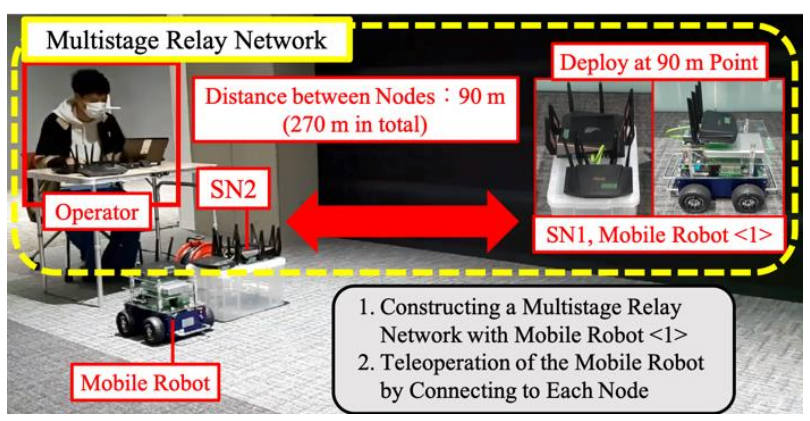

Fig. 11. Experimental Environment, Construction of Multistage Relay Network and Teleoperation of Mobile Robot.

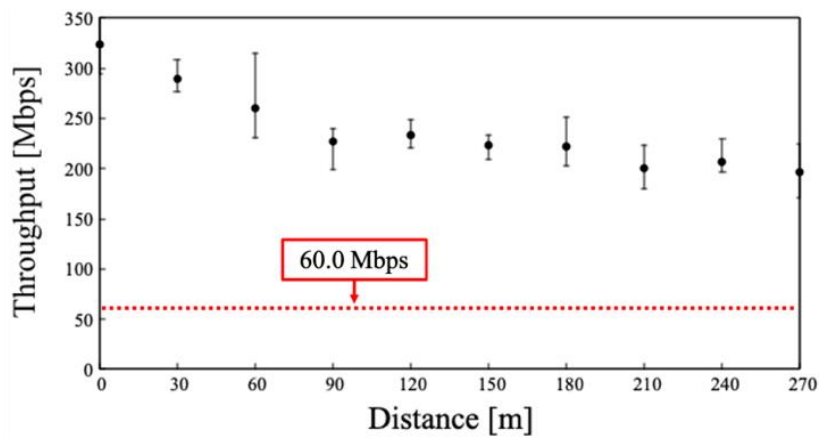

Fig. 12. Throughput of Upload between Operator PC and Mobile Robot <1> in Triple-hop Multistage Relay Network.

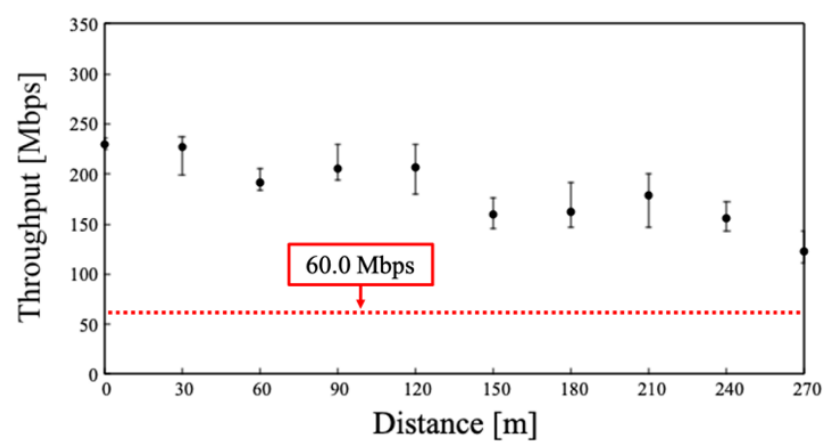

Fig. 13. Throughput of Download between Operator PC and Mobile Robot $<1>$ in Triple-hop Multistage Relay Network.

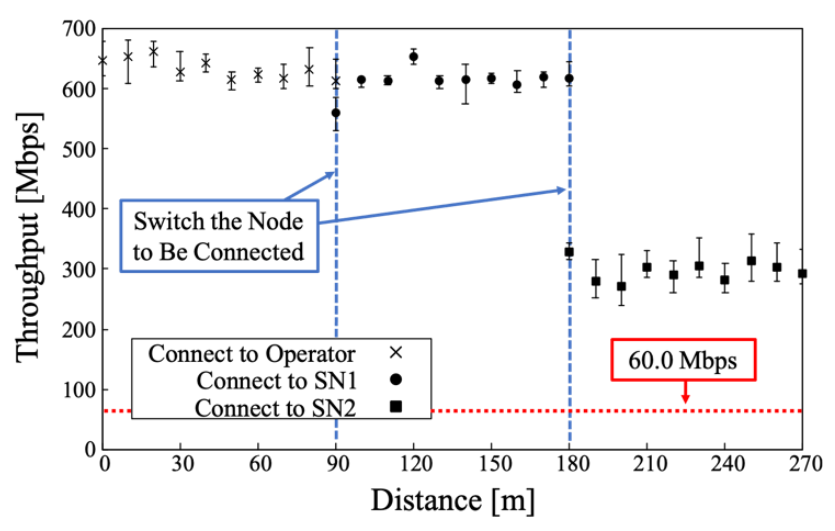

Fig. 14. Throughput of Upload between Operator PC and Mobile Robot during Mobile Robot Teleoperation.

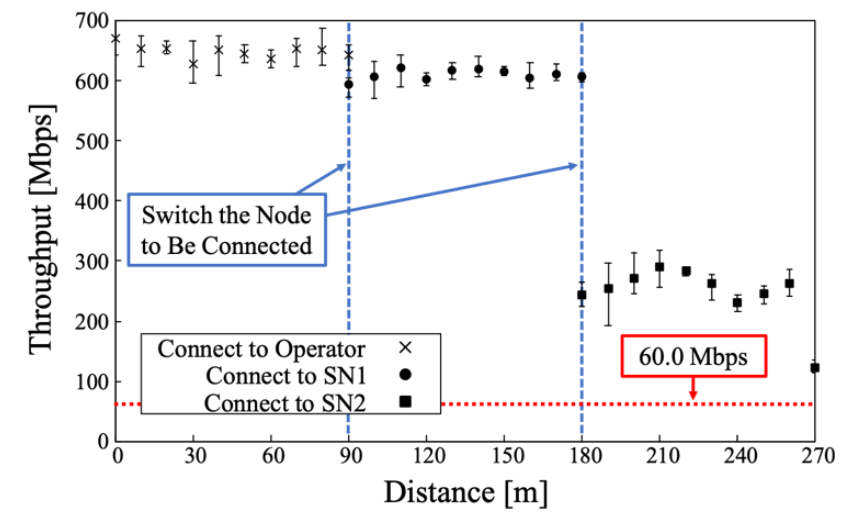

Fig. 15. Throughput of Download between Operator PC and Mobile Robot during Mobile Robot Teleoperation. 


\section{DISCUSSION}

As the distance between nodes increased, the throughput between the operator PC and mobile robot $<1>$ was measured up to the $225 \mathrm{~m}$ point. Fig. 9 and 10 show the throughput decreased by more than $100 \mathrm{Mbps}$ when the distance between nodes was about $140 \mathrm{~m}$. This decrease in throughput was deemed to have been caused by fallback, which decreases throughput in relation to a decrease in electric field strength. In the transmission and receiving of data between nodes, a delay or disconnection occurs if the amount of communicable data is exceeded. Furthermore, in wireless LANs, communication connectivity between nodes is maintained by fallback, which sets an upper limit on the transmission speed according to the electric field strength [20]. Therefore, the experiment of Section 6 assumed that the decrease in throughput at $140 \mathrm{~m}$ was due to the decrease in electric field strength caused by the increase in distance between nodes. This result suggests that it is necessary to monitor not only the throughput but also the electric field strength in a multi-robot system.

The multistage relay network was expanded to $270 \mathrm{~m}$, maintaining a throughput of more than $60 \mathrm{Mbps}$. Therefore, more than three mobile robots could be teleoperated at $270 \mathrm{~m}$ in the network constructed in this experiment. A comparison between Fig. 9 and 10, and Fig. 12 and 13 indicates that the multistage relay network provided a larger search area. Moreover, in the teleoperation of the mobile robot, a throughput of more than $60 \mathrm{Mbps}$ was maintained between the operator PC and the mobile robot after the construction of the multistage relay network. Thus, more than three mobile robots could be teleoperated when connected to any node of the network constructed in this experiment.

An RWSN cannot maintain the required throughput for multi-robot teleoperation, but the proposed method can ensure stable communication connectivity for multi-robot operation. The proposed topology is also effective in intricate environments because it can be used to construct a multistage relay backbone network with a high throughput.

\section{CONCLUSION}

This paper proposes a multistage relay network topology for constructing an environment with mobile robots for information gathering in large-scale facilities. Teleoperation of a mobile robot using a multistage relay network is effective for exploring in intricate environments and obstacle spaces due to its flexibility and scalability. However, in a multistage relay network, the communication quality decreases due to the increase in the distance between nodes and the number of relays, so a single robot is typically operated to maintain communication connectivity. As the search range of a single robot is limited, this paper considers the importance of operating a multi-robot system in a large-scale facility. A multistage relay network consisting of the proposed topology can connect multiple mobile robots to each node. These robots change the search range by switching the nodes to which they are connected. The experiment of Section 7 connected a mobile robot to a multistage relay network consisting of the proposed topology and confirmed its effectiveness by measuring the throughput. Findings indicated that this topology is effective for constructing a multi-robot environment in a large, enclosed space.

The proposed topology is effective for the teleoperation of multi-robot systems; however, there is a communication time loss when a mobile robot switches between connected nodes. Additionally, the mobile robot may become isolated if it fails to maintain the communication quality required for teleoperation in the communication path after changing nodes. Therefore, the future aim to develop a method of changing the communication route while maintaining the communication connectivity of a mobile robot.

\section{REFERENCES}

[1] Yoshiaki Kawata, "The great Hanshin-Awaji earthquake disaster, damage, social response, and recovery," Journal of Natural Disaster Science, Vol. 17, No. 2, pp.1-12, 1995.

[2] L. Ernesto Dominguez-rios, Tomoko Izumi, Yoshio Nakatani, "A disaster management platform based on social network system oriented to the communities self-relief," IAENG International Journal of Computer Science, Vol. 42, No.1, pp.8-16, February 2015.

[3] Sabarish Chakkath, "Mobile robot in coal mine disaster surveillance," IOSR Jourbal of Engineering, Vol. 2, No. 10, pp. 77-82, 2012.

[4] Keiji Sakuradani, Keigo Koizumi, Kazuhiro Oda, Satoshi Tayama," Development of a sloap disaster monitoring system for expressway operation and maintenance control," Jounal of GeoEngineering, Vol. 13, No.4, pp.189-195, December 2018.

[5] F. Kurz, D. Rosenbaum, J. Leitloff, O. Meynberg, P. Reinartz, "A real time camera system for disaster nad traffic monitoring,". https://core.ac.uk/download/pdf/11146229.pdf

[6] Jingxuan Sun, Boyang Li, Yifan Jiang, Chih-yung Wen, "A camerabased target detection and positioning UAV system for search and rescue (SAR) Purposes," Sensors 2016, Vol. 16, No. 11, 1778. https://doi.org/10.3390/s16111778.

[7] Masataka Fuchida, Shota Chikushi, Alessandro Moro, Atsushi Yamashita, Hajime Asama, "Arbitrary viewpoint visualization for teleoperation of disaster response robots," Journal of Advanced Simulation in Science and Engineering, Vol. 6, No. 1, pp.249-259, 2019.

[8] Hemanth Reddy A, Balla Kalyan, Ch. S. N. Murthy, "Mine Rescue Robot System - A Review," Procedia Earth and Planetary Science, Vol.11, pp. 457-462, 2015.

[9] Trupti B. Bhondve, Prof.R.Satyanarayan, Prof. Moresh Mukhedkar, "Mobile rescue robot for human body detection in rescue operation of disaster," International Journal of Advanced Research in Electrical, Electronics and Instrumentation Engineering, Vol.3, No.6, pp.98769882, June 2014.

[10] Zia Uddin, Mojaharul Islam, "Search and rescue system for alive human detection by semi-autonomous mobile rescue robot," International Conference on Innovations in Science, Engineering and Technology, Octorber 2016.

[11] Xuewen Rong, Rui Song, Xianming Song, Yibin Li, "Mechanism and explosion-proof design for a coal mine detection robot," Procedia Engineering, Vol. 15, pp.100-104, 2011.

[12] Tomoaki Yoshida, Keiji Nagatani, Satoshi Tadokoro, Takeshi Nishimura, Eiji Koyanagi, “ Improvements to the rescue robot Quince toward future indoor surveillance missions in the Fukushima Daiichi Nuclear Power Plant," Field and Service Robotics, pp. 19-32, December 2013.

[13] Albert Ko, Henry Y. K. La, "Robot assisted emergency search and rescue system with a wireless sensor network," International Journal of Advanced Science and Technology, Vol. 3, pp.69-78, Febuary 2009.

[14] Andrew Wichmann, Burcu Demirelli Okkalioglu, Turgay Korkmaz, "The integration of mobile (tele) robotics and wireless sensor networks: A survey," Computer Communications, Vol. 51, No.15, pp. 21-35, September 2014.

[15] Yasushi Hada, Osamu Takizawa, "Development of communication technology for search and rescue robots," Journal of the National 
Institute of Information and Communications Technology, Vol. 58, pp. 131-151, 2011.

[16] Carlos Marques, Joao Cristovao and Paulo Alvito, "A search and rescue robot with tele-operated tether docking system," Industrial Robot: An International Journal, Vol. 34, No. 4, pp. 332-338, 2007.

[17] Yuta Koike, Kei Sawai, Tsuyoshi Suzuki, "A study of routing path decision method using mobile robot based on distance between sensor nodes," International Journal of Advanced Research in Artificial Intelligence, Vol. 3, No. 3, pp. 25-31, 2014.

[18] Kei Sawai, Ju Peng, Tsuyoshi Suzuki, "Throughput Measurement Method Using Command Packets for Mobile Robot Teleoperation Via a
Wireless Sensor Network," (IJACSA) International Journal of Advanced Computer Science and Applications, Vol. 7, No. 4, pp 348- 354, 2016.

[19] Tsuyoshi Suzuki, Ryuji Sugizaki, Kuniaki Kawabata, Yasushi Hada, Yoshito Tobe, "Autonomous deployment and restoration of sensor network using mobile Robots," International Journal of Advanced Robotic Systems, Vol. 7, No. 2, pp. 105-114, 2010.

[20] Jeba Sonia J, Julia Punitha Malar Dhas, "A rate adaptation algorithm for IEEE802.11 wireless networks for commercial applications," Journal of Chemical and Pharmaceutical Sciences, Vol. 9, No.4, pp. 1904-1908, 2016. 